\title{
COLLABORATION AND ALTERED PROCESSES
}

\author{
SEAN AHLQUIST \\ School of Architecture \\ California College of the Arts \\ 1111 Eighth Street, San Francisco, CA 94107-2247, USA \\ sean@proces2.com
}

\begin{abstract}
The architectural technologist could be defined as a designer whose methods are driven by the intimate and experimental use of varying digital technologies. The technologist, in this scenario, is a designer by training, but typically, seen primarily for their technical expertise. Through an emerging practice of the architectural technologist as a design collaborator, an identity is forming of the technologist as a designer who balances general issues of architecture with an analytical mind towards digital/computational methods. Collaboration exists ultimately in the realm of design (not in production) but introduces a shift in process, where design involves the construction of digital means and the critique of process by all participants in the collaboration. This paper describes the necessity of this type of collaboration in relation to several specific design projects, to which Sean Ahlquist / Proces2 participated as the technologist. At issue is the technologist's degree of influence on the processes, the level of exchange between designers, and the resulting influence on the success of the design. Three projects will be discussed to show the range of collaborative interaction. In one scenario, the technologist worked within a stratified and somewhat traditional process based on the applying digitallyderived systems to a specified form. A second scenario looked to find a generative, computational method through the collaboration. The intent was to discover an architectural pattern that had an advanced level of complexity, and simultaneously provide data for fabrication and construction. The last project saw the collaboration as a necessity to produce an array of highly complex 3-dimensional forms and provide means of communication between the highly digital environment and analog means for analysis and fabrication.
\end{abstract}

\section{Introduction}

In collaboration with an architectural technologist, design exists within the critique of both process and product. Design is validated in the logic of the process and its relevance to the performance of the final output. Within the eyes of the architectural technologist, the ultimate desire is to completely merge the relationship between a digital / generative process, and performance of the product, as well as its fabrication, balancing consideration for both design and process. In the projects described in this paper, the technologist would be considered an active participant, but with different levels of influence within the design process and to the final outcome of the design. The topics of each project differ considerably, but in the exchange between the designer and design technologist, there is a noticeable shift in methods of communication and processes that generate the architecture. Consistent in this shift, though, 
is the significance of digital, systematic processes as a mechanism to both conceptualize and realize the design.

\section{Applied Geometries}

The Jellyfish House ${ }^{1}$, designed by IwamotoScott Architecture with Proces2, was created through a relationship where the architectural technologist was necessary in satisfying a digital component within a multi-faceted and largely theoretical project. The project looked to describe an architecture based on distributed systems, and networks. The technologist, in this case, was not so much invested in the derivation of the concept, but more so in the design and discovery of the digital methods that could geometrically express the concept. The process was separated between studies of planning at an urban scale, refinement of form at the local (dwelling) scale, and application of the skin/structure system to the individual dwelling. Through the linear process, collaboration was shifted from being an inclusive exchange of ideas between each level of the project, to determining the best means of communication and translation between the different stratified levels of the design process. The process delivered a geometrically complex design, but only with the technologist as a thin layer within a hierarchical design approach.

\subsection{Project description}

The Jellyfish House is a theoretical proposal designed for the exhibit "Open House: Architecture and Technology for Intelligent Living". The exhibit hosted a series of projects that consider emerging technologies, digital and material, and how they may influence the design and nature of a future domestic environment. The proposal looked to the Jellyfish to develop a notion about an architecture that can function as a series of distributed, adaptive, and sensing networks. These networks apply to various conditions of the environment (both internal and external) and factors of domestic infrastructure (structure and mechanical systems). Performative elements are built within a singular surface that wraps the entirety of the house and also links to the surrounding landscape. The surface is a thickened structure, a "deep surface" that acts as the sole means for housing all of the systems, both transforming and fixed.

\subsection{Process}

IwamotoScott uses a process of research into materials, technology, and organizational logics to develop work that investigates perceptual and experiential phenomena. With the Jellyfish House, the initial impetus was to add into the process a higher level of digital experimentation, one at the level of complex digital modeling. Specifically, work that was previously done by Proces2 in studying cloth dynamics with Delaunay ${ }^{2}$ and Voronoi ${ }^{3}$ tessellation methods was layered into the concept about distributed and integrated networks. These two methods of tessellation were applied to the form of the overall house as a network / structural skin. The form of two twisted intertwining boxes was central to the design. The critical nature of the process came in developing the logic for the pathway that applied the skin geometries to the form of the house. The necessity in collaboration was in communicating between designers in a language of both design and digital modeling technology.

The overall method worked in three somewhat distinct modes, executed in a linear fashion, and challenging the notion of design communication and feedback. The complexity of the desired geometric pattern demanded that several softwares be used. Translation between softwares exacerbated the linear nature of the process and shifted the discussion from commentary about design to challenging the fluidity of the process. Models and data were translated between software such as Rhino, Autodesk MAX, Excel, and GenerativeComponents ${ }^{4}$. The nature of the process forced a shift in what is defined as feedback for analyzing the design. In a more traditional process, design is developed and refined through viewing iterations of the final outcome. The complexities of the process, in this instance though, meant studying the design through analyzing the product at each step of the process, and viewing snippets of the final outcome. Critique was of the logic of process and design established at each step. Again, it was necessary that collaboration meant discussion of technique not just of the outcomes. 

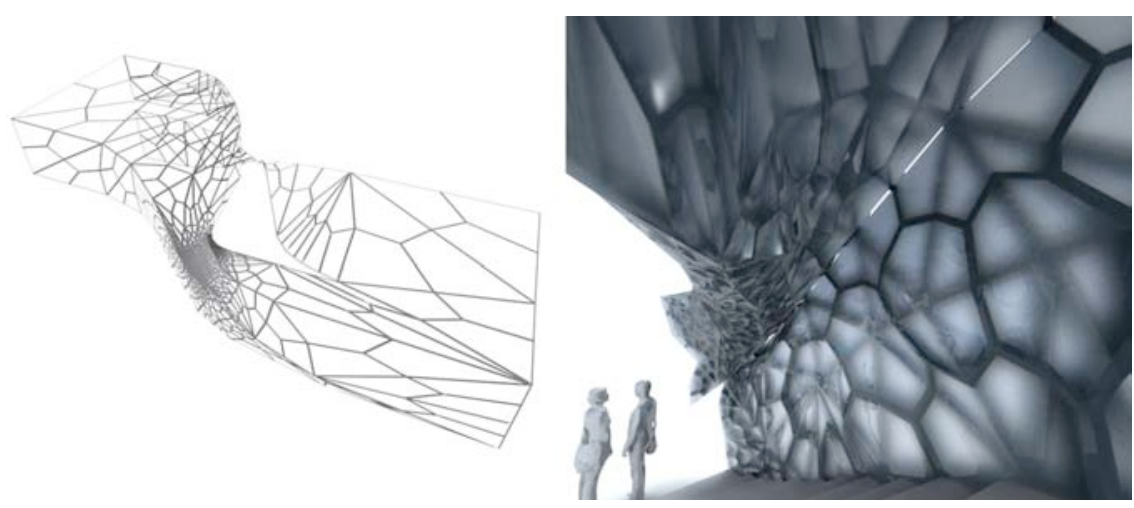

Image 1: Delaunay / Voronoi tessellation and sample region of parametric component model

The connection between the Delaunay / Voronoi tessellation and the concept of distributed networks expressed a condition where process and concept were intended to be seamless. The Delaunay algorithm in MAX subdivides a surface where advanced curvature demands higher subdivision. In the spatial planning of the house, the moments of higher subdivision corresponded with areas that needed density in the network. As the method of skin definition and shaping of the overall form were on distinct layers of the process, exploiting this connection between process and concept was limited. Surface curvature was a formal device for expressing a series of continuous spaces. The tessellation was a pattern applied to the form. It was a formal expression as opposed to an adaptive, influential condition. For the process to inform and advance the design, the collaborators had to bridge the design intents with the details of the digital tools. For the technologist, the issue laid in designing the appropriate and logical variables for controlling the tessellation / skin system. For IwamotoScott, understanding the logic of the variables was necessary for guiding the manipulation of the form. With limited exchange between different levels of the design and amongst the tools that drive the design, the desired product was always elusive. This moment exemplifies the struggle between a digital, systematic approach, and a linear, formalistic approach where image and result outweigh the product born intimately of method. The split in design approach was evident in the thinness of the skin; its minimal responsiveness and investment into the formal logic of the massing.

\section{Generated Complexity}

The Airspace Tokyo ${ }^{5}$ project, a collaboration of Thom Faulders with Proces2, describes a relationship where the technologist was embedded into the experimentation and generation of the conceptual and physical implementation of the design. There was not a significant shift in process for the collaboration rather a recognized need for computational methods to achieve the conceptual intention. The intention for the design was to generate an architectural pattern that had a depth in complexity along with a component of unexpectedness. This spoke to a generative method of design where the outcome was an aggregation of both controlled and uncontrolled conditions. While virtual, in the sense that the process lived within the digital realm, the product was always seen as architectural where conditions could be controlled to respect materiality and the product was always usable for fabrication of the screen itself. The situation expressed compatibility between technologist and collaborative designer, one where automation in design process was accepted and desired.

\subsection{Project description}

The design is for an architectural screen that wraps a 4 story residential building in Tokyo. The impetus for the design was to create a narrow buffer zone around the building, protecting it from the intense urban environment immediately adjacent. Previously, a thick band of vegetation bordered the site, and had provided such a shield. The new building allows for a slight $30 \mathrm{~cm}$ depth for the architectural screen. While narrow in section, the screen intends to engage the natural and urban conditions. The screen mediates wind, diffuses and reflects light along it surfaces, and acts a light rain screen for the adjacent walkways and balconies in the building. Views inward are mediated by the shifting variegated cellular pattern. The system needed to have a depth and effect to where moving through and along it presented a shifting and everchanging perception of the pattern. Much like a tree canopy or the structure of a sponge, 
different angles of the architecture provide different expressions of the system, in this case, a layering of voids and shifting views.

\subsection{Process}

Faulders work places an emphasis on research and application. His designs view architecture as a responsive and dynamic medium, looking to both digital and materials systems to explore such realities. With the Airspace Tokyo project, digital technology was pursued to create the means for generating an advanced level of complexity in the pattern of the screen, and directly providing the architecture for the project, the data for construction of the screen. From the outset, it was an experimentation in method, but one that demanded algorithmic and scripted procedures. It was desired that the method be clear and generative; where the results of it being obvious in its systematic nature but devious in revealing itself, not easily discernable. This was accomplished through layering variation into the system, and layering the very system back onto itself. There was a desire for the outcome to be somewhat unexpected, and an acceptance that no two iterations of the process would produce the same result. The slight shift in process was a willingness to trust the method, let the system aggregate, as designed, impose controls where necessary and produce a highly systematic, complex pattern. In collaboration, both designers engaged the details of the computational method. But, constructing the series of algorithms and computations to produce the design was left within the hands of the technologist. Where the product was generative, it was necessary and allowable to have this level of autonomy within the process. Collaboration succeeded where both parties understood the affects of the computational variables to the result, but the construction and mechanics of the digital functions where left solely to the technologist.

To produce the balance of systematic and unexpected results, the method layered together a series of modeling and computational procedures. Pairs of patterns were generated from the process and subsequently compressed into a single layer, producing a higher level of complexity and unpredictability. To produce a single pattern, an intricate polygonal framework was created, using tools of subdivision and tessellation in Autodesk MAX. Exposing variables with the meshing algorithms, varying levels of subdivision were generated in loose recognition of the building programming. A cellular, parametric component, constructed in GenerativeComponents, was then aggregated throughout the framework. To produce a higher order of complexity, two layers of cellular panels where compressed into a single skin. Welding the two layers together produced additional orders of the system where new shapes of apertures emerged, beyond the basic open-cell construct. To add a layer of control within the system, computations were added to the component responding to basic material assumptions, limiting the thinness of the pattern at certain moments to ensure stability. Design was in developing the set of computations and variables that allowing tuning of the balance between unpredictability and control.

Compared to the initial study of foliage, we saw a system where the logic could be mathematically described, but the perception was of something unpredictable. This was a foil for both the method, and the product of the method. The tools were manually constructed as a set of commands, variables, and hierarchical relationships. When executed, it produced a system that could not be manually generated or exactly predicted. This expressed a process that was altered to weigh towards a trust of the generative method and a desire for its unexpected results.

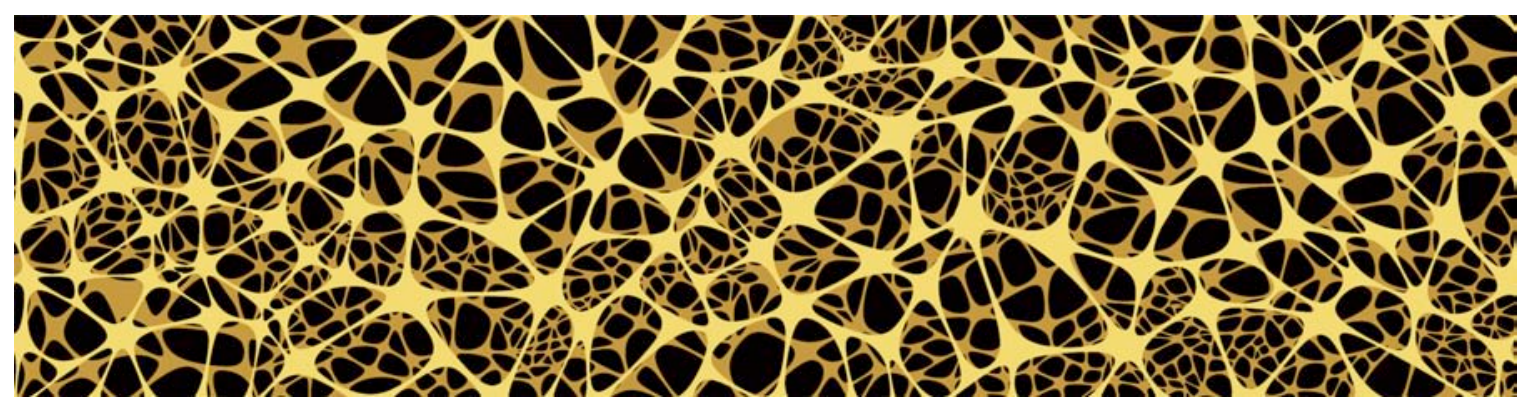

Image 2: Individual layers to be compressed to create single skin 


\section{Parametric Complexity}

With the Kinetic Sculpture ${ }^{6}$ project, designed by Reed Madden Designs, the role of the technologist was primarily as a problem-solver. The focus of the digital work was to rationalize the design, ensure its buildability, and develop and provide all necessary information for fabrication. The design was simple in concept, where the complexity laid in the nature of the curvature of the forms and the interrelationships between them. It was apparent that pursuing a digital model was the only way to resolve issues of understanding the design at full scale. The sculptors looked directly to the physical to judge and compose the design. Working within a highly digital environment, that way of perceiving design significantly shifted and adapted to a language that dealt with digital geometries and associative parametric models. From the technologist's perspective, a balance had to be struck between satisfying the sculptor's desire for an expressionistic, interpretative sculpture and following the inherent associative geometric rules that the forms clearly represented.

\subsection{Project description}

The project is a public sculpture for the center of downtown Tiburon in Northern California. The sculptors developed the design to make reference towards the sails of the boats docked nearby and the ocean life itself in the neighboring bay. The sculpture is built of 5 independent metal fins. They are designed so that they nest in a closed position to resemble a shark's dorsal fin in profile and, loosely, in surface curvature. When open, the sculpture resembles five sails catching wind. The sculpture both conceptually and for purposes of fabrication required that the relationships of curvature across the array of sails be clearly geometrically defined and highly controllable.

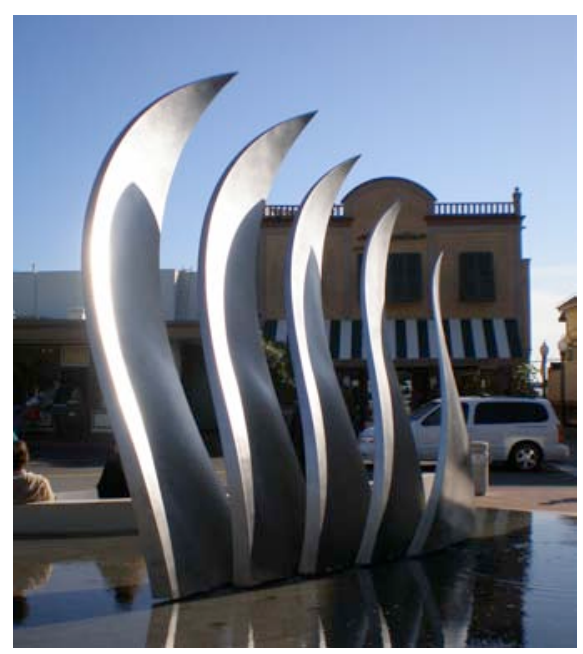

Image 3: Completed kinetic metal sculpture

\subsection{Process}

The sculptors work focuses on creating site specific, public sculptures. With the Tiburon kinetic sculpture, they found that using digital models was the only way to generate their design with accuracy at full scale. The design pursues the artist's interest in composing the line against the curve. The challenge as they saw it was to not just compose, but to also composite the two into a singular form, the ruled surface. But, as what has always been a limitation, the constraints of the metal material make constructing these forms elusive. The initial impetus was in simply understanding the nature of the forms they proposed. In the artist's eyes, the expression and metaphor of the sculpture was primary. For the technologist, in this case, the definition of the geometric rules and the adherence to those rules was what defined the project.

This system of forms represented a clear definition of a parametric associative model. There was no one fin that represented the ideal profile and shape. It was only in seeing all of the forms that the degrees of curvature and relationships from one to the next could be verified. When hierarchically all values are equal, it presented a problem in determining where to begin. In this case, the elevational study of the fin profiles, individually and as a whole, was the first 
step at establishing the parametric relationships. What became the solution was defining a type of a curve that was composed of various base geometric elements and blends between them.

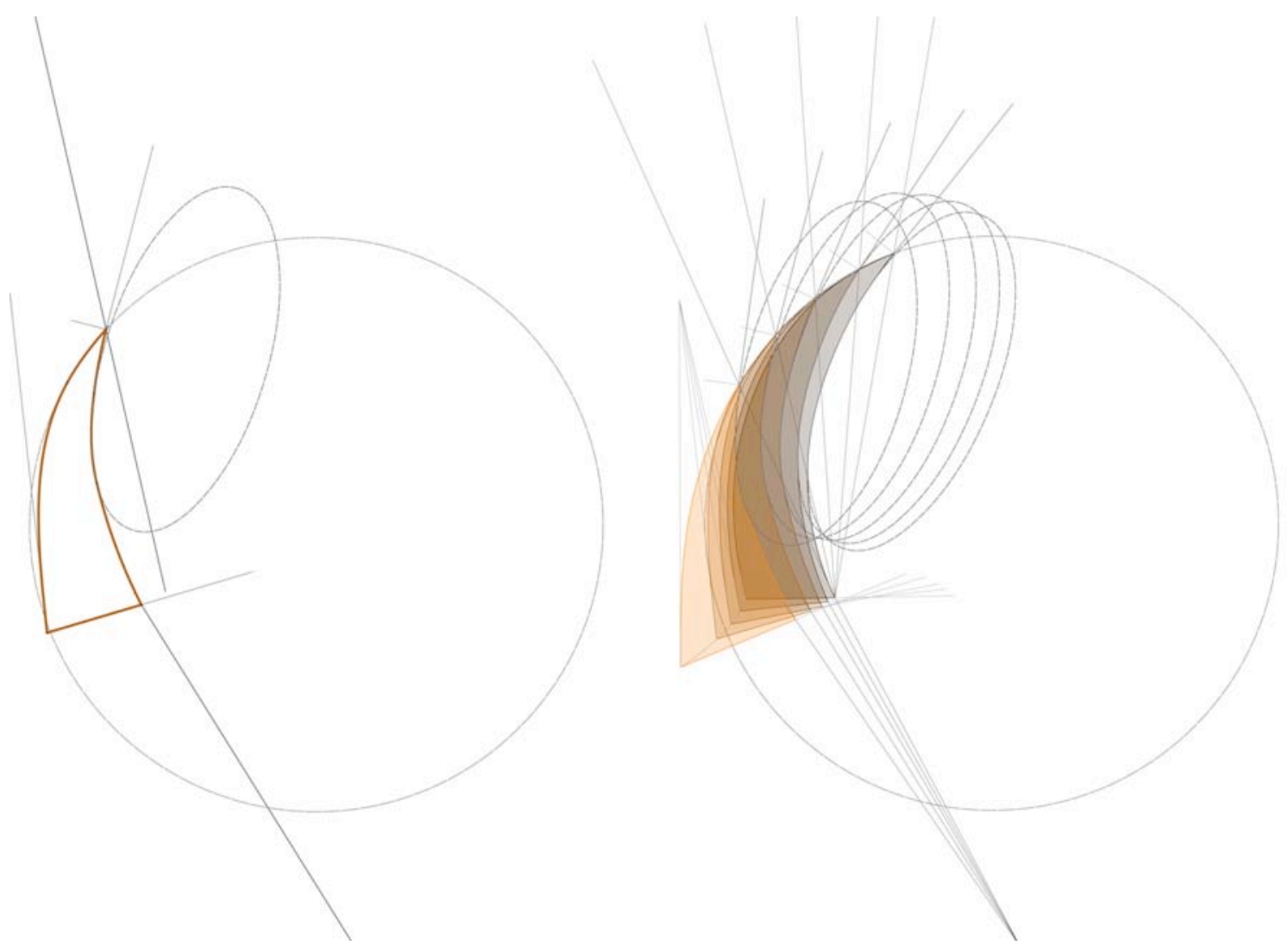

Image 4: Parametric drawings used to define elevational profile of sails

The scale and rotation of these base elements were the variables, and the portion of the line that was sampled for the fin was also a shifting parameter. An additional series of curve types was generated to form the three dimensional curvature of the forms. At the same moment of satisfying the associative relationships, the requirement for ruled surfaces had to be met. For the artists, the digital model was both enlightening and frustrating. At one moment, they analyzed the very detailed curvatures, but at another moment they could not enter into the direct manipulation of it, nor look to localize the adjustments, for it would break the associative model. It speaks to the contrast between a desire to be systematic and process driven, or driven by the analysis of the final product. Here the process is trying to mediate both, where criticism of the form challenges the tools of the process. The tools are not informing the design but being refined and further understood to satisfy the ultimate concept of a series of cooperatively curving forms.

\section{Conclusion}

In the simplest definition of collaboration, all participants have equal interchange of ideas and influence acts in a decentralized manner, rather than hierarchical. Collaboration with an architectural technologist is intended to act in the same manner. In terms of architectural design, this type of specific collaboration does propose a shift, though, where a priority lies in the logic of process and the specificity of the digital tools utilized for design. Collaboration becomes a discussion of design issues and digital methods. With the Jellyfish House, collaboration and process were hierarchical. Communication about digital procedures was minimized. The outcome was affected where all participants of the project were not engaged and sufficiently knowledgeable in the digital tools necessary to produce the design. The modeling technology was necessary as a component of the design. But without proper comprehension of the tools and variables of that technology, that design component could not be fully expressed, engaged, and controlled. With the Airscape Tokyo and Kinetic Sculpture projects process had an inextricable link to the product of the design. Collaboration was in critique of the product and the process. With both projects, there was a pursuit of the 
architecture, where process was not in depicting the design but in generating digital information that related to fabrication and construction. Airspace Tokyo engaged technology in process to produce computationally-driven, geometrically complex results. The Kinetic Sculpture pursued technology to rationalize the geometric complexity of the forms, while also maintaining their ability to be formed from single sheets of material. In collaboration, there was simultaneity in critiquing process and design, and understanding the direct relationship between them.

Without a clear and even exchange at the levels of process and product, the engagement of the technologist as a contributing designer will be minimized. Any design collaboration will take on this same dynamic. For the technologist, the philosophy and practice has to be balanced between general issues of design and the expertise of digital methods. In collaboration, process is more exposed and therefore the communication must exist at that level. With a constant desire to engage technology to absorb its generative and advanced capabilities, there is a challenge to simultaneously participate in the language of the process and tools, and address the architectural intentions of the design.

\section{Notes}

1 Jellyfish House. IwamotoScott Architecture w/ Proces2. 2006. Project commissioned for the "Open House: Architecture and Technology for Intelligent Living" exhibit curated by Vitra Design Museum and Art Center College of Design.

${ }^{2}$ Delaunay is a triangulation method where the minimum angle of all the angles of a triangle is maximized, this tends to prevent "sliver" triangles. This method also forces the triangles to be somewhat equilateral. In Autodesk MAX, Delaunay is used as a subdivision method for NURBS surfaces. It is often selected for the subdivision of surfaces being used for cloth dynamics. The Delaunay triangulation provides for the most accurate results when calculating cloth dynamics.

${ }^{3}$ Voronoi is a tessellation method such that each polygon contains exactly one generating point and every point in a given polygon is closer to its generating point than to any other. Autodesk MAX uses a simulated version of the Voronoi method as a subdivision method with its MeshSmoothing modifier.

${ }^{4}$ GenerativeComponents is a parametric and associative modelling software developed by Robert Aish and Bentley Systems.

${ }^{5}$ Airspace Tokyo. Building design by Studio M, Hajime Masubuchi. Architectural screen design by Thom Faulders with Sean Ahlquist / Proces2. Tokyo, Japan. 2007.

${ }^{6}$ Kinetic Sculpture titled "Coming About". Reed Madden Designs with Sean Ahlquist / Proces2. Tiburon, California. 2006. 